ÖZS 34 (2009) 4:3-12

DOI: $10.1007 / 10.1007 / \mathrm{s} 11614-009-0037-0$

EDITORIAL $\quad$ i

\title{
Zwischen „Weltklasse“ und „Nomaden wider Willen"61 - Soziologische Beiträge zur Globalisierung des Managements
}

\author{
Ursula Mense-Petermann
}

„Management am Scheideweg?““, „Neue Werte in den Führungsetagen?“, „Die Leistungsträger" - die Titel aktueller Tagungen und Publikationen ${ }^{2}$ zeigen es an: Nachdem die Gruppe der Manager lange Zeit kaum im Zentrum von (arbeits-, wirtschafts- oder organisations-)soziologischen Debatten stand, steht sie nun in der Folge von Finanz- und Wirtschaftskrise nicht nur in Medien und Öffentlichkeit, sondern auch in der soziologischen Debatte ganz oben auf der Agenda. Auch das hier vorliegende Themenheft widmet sich einem managementsoziologischen Thema. Gefragt wird nach aktuellen Entwicklungen im Management im Kontext von Globalisierungsprozessen.

In der Debatte um ökonomische Globalisierung waren es allerdings zunächst die so genannten transnationalen Unternehmen, auf die sich die Forschung konzentrierte. Als „treibende Kräfte“ wirtschaftlicher Globalisierung (Altvater/Mahnkopf 1997), als die zentralen Akteure in Prozessen weltwirtschaftlicher Vergesellschaftung (Zündorf 1999), als wichtige Eigenstrukturen von Weltgesellschaft (Stichweh 2001) und als organisatorischer ,Idealtyp“ im Umgang mit höchst komplexen und global konstituierten Umwelt- und Wettbewerbsbedingungen (Bartlett/Ghoshal 1990) stehen transnationale Unternehmen im Zentrum unterschiedlicher theoretischer Ansätze in der Globalisierungsdebatte. Hier wird transnationalen Unternehmen als weltwirtschaftlichen Akteuren viel ,zugetraut ${ }^{*}$ - Probleme, Widersprüche und vor allem auch ein mögliches Scheitern der Umsetzung von Transnationalisierungsstrategien blieben in diesen Makroperspektiven allerdings zunächst unterbelichtet. In jüngerer Zeit hat es denn auch eine Reihe von kritischen Anfragen an die Bedingungen der Möglichkeit der Stabilisierung transnationaler Koordinations- und Organisationsformen gegeben (vgl. etwa die Beiträge in Mense-Petermann/Wagner 2006). Dabei richtet sich die Aufmerksamkeit auch zunehmend auf die Manager transnationaler Unternehmen als zentralen Trägern von Transnationalisierung.

Erste ,starke' Thesen zur Globalisierung des Managements stammen aus der amerikanischen Managementforschung. Hier wird der so genannte ,Globalmanager' als neuer, zur globalen Wirtschaft komplementärer Managertypus postuliert. Der ,Globalmanager ‘ repräsentiert den Idealtypus des Managers von transnationalen Unternehmen, der für die Anforderungen sich globalisierender und immer komplexerer Umwelten von global players bestens gerüstet ist (Bartlett/Ghoshal 1990; Kanter 1996). „Die Entwicklung multidimensionaler Management-Perspektiven und -Fähigkeiten erlaubt ihnen, Chancen und Gefahren überall in der Welt zu erkennen und auf Veränderungen rasch und angemessen zu reagieren.“ (Bartlett/Ghoshal 1990: 222; vgl. auch Bossard/Peterson 2005). Insofern spielt der ,Globalmanager' für die transnationale Koordination von 
Wirtschaftsaktivitäten eine zentrale Rolle: Eine Matrix-Struktur zu entwickeln - so lässt sich die Management-Literatur zusammenfassen - ist eine notwendige, aber keineswegs hinreichende Bedingung des erfolgreichen Umgangs mit höchst komplexen, global konstituierten Umwelten. Wichtiger noch ist für diese Autoren die ,Matrix im Kopf ${ }^{\star}$ der ,Globalmanager', also die Entwicklung einer multidimensionalen und interkulturellen ,Haltung'.

Ein anderer Zweig der amerikanischen Managementforschung thematisiert Globalisierungsprozesse im Management unter dem Aspekt von Managerkarrieren. Diese Debatte wird beherrscht von den komplementären Thesen der ,, boundaryless career “ (Arthur/Rousseau 1996) und „protean career“ (Hall 2004). Beide Thesen behaupten für Karriereverläufe eine Umstellung von der Organisation und der in ihr und von ihr institutionalisierten Karrieremuster auf den einzelnen Manager und seine individuellen Strukturierungs- und Managementleistungen. Typisch für das bürokratische Großunternehmen der Fordistischen Ära war die so genannte Kaminkarriere: Diese bezeichnet den positionalen Aufstieg in der Hierarchie einer einzigen Organisation, also eine Hauskarriere. Die Organisation spielt für diese Karriereform eine zentrale Rolle: Organisationale Karrieresysteme definieren die Möglichkeiten der individuellen beruflichen Entwicklung, indem sie mögliche Aufstiegswege und Karrieremuster und die Zugangsbedingungen vorgeben. Die beiden komplementären Thesen der boundaryless und der protean career legen nun ein Ende dieser Karriereform nahe. Hintergrund dafür ist vor allem die Abkehr vom bürokratischen Organisationsmodell mit seiner tiefgestaffelten Hierarchie und entsprechend zahlreichen Aufstiegspositionen als organisationalem Kontext der Kaminkarriere. Auslandseinsätze und häufigere Organisationswechsel über nationale Grenzen hinweg gelten dann als wichtige ,Bausteine‘ für den individuellen Aufbau von Karrieren.

Anknüpfend an diese Thesen legt die Harvard-Ökonomin Rosabeth Moss Kanter mit ihrem Buch „Weltklasse“ eine noch weiter gehende sozialstrukturelle These vor: Sie geht von der Herausbildung einer globalen Klasse von, Globalmanagern “ und von einer Verschmelzung der Managementkulturen im Topmanagement zu einer, Weltkultur des Managements' ${ }^{6}$ uus. $^{3}$

Trotz des normativen Duktus solcher Beiträge aus der internationalen Managementforschung ist die These vom ,Globalmanager' und der Herausbildung einer transnationalen Wirtschaftselite auch soziologisch interessant. Die soziologische Globalisierungsforschung hat Globalisierungs- und Transnationalisierungsprozesse im Bereich des Managements allerdings erst mit einiger zeitlicher Verzögerung zu ihrem Thema gemacht. In jüngster Zeit hat jedoch das Interesse soziologischer Beobachter an Globalisierungsund Transnationalisierungsprozessen im Bereich des Managements zugenommen. Aus drei Richtungen liegen hier Beiträge vor, die sich zumeist kritisch mit der ,Globalmanager'-These auseinandersetzen:

In der Elitenforschung hat etwa Michael Hartmann die ,Globalmanager'-These in mehreren empirischen Studien $(1999,2007)$ zur Internationalisierung des Topmanagements von global players auf den Prüfstand gestellt. Anhand der Ausbildungs- und Karriereverläufe der Top-Manager der größten deutschen, französischen, britischen und US-amerikanischen Unternehmen ist er der Frage nachgegangen, „ob von einer solchen internationalen ,business class' bereits gesprochen werden kann und, falls ja, wieweit 
der Prozess ihrer Bildung vorangeschritten ist“"(Hartmann 1999: 116). Hartmanns Fazit: Die Internationalisierung der Wirtschaft habe die nationale Prägung der Topkarrieren in den Großkonzernen bislang kaum verändert, die Dominanz der nationalen Aufstiegspfade in Spitzenpositionen der Wirtschaft sei ungebrochen (ebd.). Auch in seiner neuesten Studie zur Europäisierung von Eliten stellt er fest, „dass von einer Europäisierung der Wirtschaftseliten allenfalls ansatzweise gesprochen werden kann.“ (Hartmann 2007: 211).

Allerdings - so ließe sich gegen Hartmanns Studien einwenden - ist eine Perspektive, die nur das Top-Management der größten transnationalen Unternehmen in den Blick nimmt, zu eng. Hier geraten Transnationalisierungsprozesse im Management unterhalb der Vorstandsebene in der nachwachsenden Managergeneration nicht in den Blick, und sie blendet damit empirische Phänomene der Ausbildung transnationaler Orientierungen in Organisationen mit möglicherweise sozialisatorischen Effekten für zukünftige Elitenformierungsprozesse aus. Die Stichhaltigkeit der ,Globalmanager'-These erweist sich nicht (nur) an den in den Nachkriegsjahrzehnten ausgebildeten und aufgestiegenen heutigen Top-Managern an der Spitze von global players, sondern (auch) an aktuellen Prozessen der Managemententwicklung im Kontext der Transnationalisierung von Unternehmen.

Eine zweite Richtung, aus der in jüngster Zeit ebenfalls Beiträge zu Transnationalisierungsprozessen im Management kommen, ist die Migrationsforschung. Transnationale Mobilität - nicht mehr nur von Arbeitsmigranten und Flüchtlingen, sondern auch von Hochqualifizierten - ist ein noch junges Forschungsfeld (vgl. Kreutzer/Roth 2006). Theoretisch interessiert sich die neuere Migrationsforschung für das Entstehen transnationaler Sozialräume (Pries 2008; Faist 2003): ,Unterhalb“ von makrosoziologischen Theorien der Weltgesellschaft wird in dieser Perspektive nach den konkreten grenzüberschreitenden Verflechtungen und Sozialbeziehungen gefragt. Es geht hier um die Frage nach neuen Beziehungen zwischen Raum und Sozialität. Inwieweit werden durch die Globalisierung des Managements solche neuen transnationalen Sozialräume aufgespannt? Die Forschungslage ist hier jedoch, was die ,Migration“ von Hochqualifizierten und Managern angeht, noch sehr dünn.

Drittens schließlich haben auch in einer arbeits- und organisationssoziologischen Perspektive Fragen nach der räumlichen Mobilität von Managern vermehrt Aufmerksamkeit gefunden. Empirische Studien über sogenannte Expatriates - Manager aller Ebenen, die auf Zeit an einen ausländischen Standort entsandt werden - haben zu Fragen darüber geführt, inwieweit sich (auch) mit der Globalisierung die Rahmenbedingungen für Laufbahnen und Karrieren verändern. Solche Studien liefern Hinweise darauf, dass sich auch im Bereich des Managements - und nicht nur für die rank and file-Mitglieder von Unternehmen - die Erosion stabiler Erwartungen und Garantien mit Blick auf berufliche Entwicklungsmöglichkeiten beobachten lässt. Damit ist das Thema anschlussfähig etwa an die Studie von Boltanski/Chiapello (2003) zum „Neue(n) Geist des Kapitalismus“, also an die These einer zunehmenden Projektförmigkeit von Arbeit und Management und dem zunehmenden ,Ausgesetztsein“ gegenüber Marktprozessen. Die Literatur zum Expatriate-Manager zeigt allerdings ein zwiespältiges Bild: Während die Business-Literatur, die das Forschungsfeld bis heute dominiert, häufig normativ von der funktionalen Notwendigkeit global mobiler, interkulturell kompetenter und zur transnationalen Ver- 
netzung fähiger Manager für transnationale Unternehmen auf deren problemlosen Einsatz schließt und dann doch auftretende Probleme mit organisationalen Defiziten bei der Rekrutierung, Vorbereitung und Begleitung der Expatriates erklärt (vgl. Black et al. 1992; Bossard/Petersen 2005), verweist die - allerdings bisher noch sehr dünne - wirtschafts- und organisationssoziologische Literatur ad Expatriates vor allem auf die Probleme und Schwierigkeiten solcher Einsätze (vgl. Mense-Petermann 2005; Wagner 2006; Klemm/Popp 2006).

Vor dem Hintergrund dieses Forschungsstands zu Fragen der Globalisierung des Managements ist es das Ziel des vorliegenden Themenheftes, die empirische Basis für die Debatte um Globalisierungsprozesse im Management zu verbreitern und die Fragen, die bislang dominant in der praxisnahen Managementforschung und dort zumeist in Form von empirisch kaum gedeckten, ,forschen' Thesen oder normativen Präskriptionen diskutiert werden, in einer soziologischen Perspektive zu diskutieren.

Eine genuine Managementsoziologie, die die „im sozialen Raum definierte Chance zur Entfaltung von Macht, Autorität und Prestige, die unterschiedlichen Weisen, in der diese genutzt werden (. . ), wie die Möglichkeiten zur gesellschaftlichen Reproduktion dieser Chance und die Rückwirkungen ihrer Realisierung (durch spezifische Personengruppen) auf die Strukturen des sozialen Raums" (Pohlmann 2002: 239 f.) untersucht, ist zwar als eigenständige soziologische Teildisziplin - trotz einzelner einschlägiger Beiträge (vgl. Faust et al. 2000; Schmidt et al. 2002; Mayrhofer et al. 2002; Kotthoff 1997; Kotthoff/Wagner 2008) - im deutschsprachigen Raum bislang nicht entstanden. Nach wie vor - und insbesondere, was die Frage nach der Globalisierung des Managements angeht - wird die Managementforschung von praxis- und beratungsnahen, häufig normativ aufgeladenen Beiträgen dominiert, und mystifizierende und heroisierende Perspektiven auf die Gruppe der Manager sind in der Managementforschung weit verbreitet. Das vorliegende Themenheft zielt demgegenüber im Sinne einer Managementsoziologie auf die empirische Beobachtung aktueller Wandlungsprozesse im Management im Kontext wirtschaftlicher Globalisierung. Es geht in dem vorliegenden Themenheft nicht um Präskriptionen für ,gutes Management', sondern um die Analyse von sich wandelnden gesellschaftlichen und organisationalen Rahmenbedingungen des Managements sowie umgekehrt um die gesellschaftlichen Folgen von Wandlungsprozessen im Management.

Alle Beiträge dieses Themenhefts nehmen die These vom ,Globalmanager' und von der ,grenzenlosen' Karriere zum Ausgangspunkt einer kritischen, empirisch gestützten Auseinandersetzung und Analyse. Sie nehmen dabei - anders als die Elitenforschung nicht das Top-Management der global players in den Blick, sondern untersuchen die transnationale Mobilität (Pohlmann/Bär; Minssen), die (interkulturellen) Erfahrungen und Sozialisationsprozesse im Zuge von Auslandseinsätzen (Minssen; Gottwald/ Klemm; Kels/Vorbusch), die Einbettung solcher Auslandseinsätze in die Berufsbiographien und Karrieren sowie die lebensweltlichen Bezüge der und die Ansprüche an die Gruppe der Nachwuchsmanager und mittleren Fach- und Führungskräfte (Kels/Vormbusch; Minssen) auf der Basis eigener empirischer Studien.

Markus Pohlmann und Stefan Bär gehen in ihrem Beitrag der Frage nach, ob sich im Einklang mit der ,starken' Globalisierungsthese die Herausbildung globaler Arbeitsmärkte für Hochqualifizierte und transnationale Karrieren im Sinne eines häufigen 
Wechsels zwischen Organisationen und über nationale Grenzen hinweg beobachten lässt. Sie knüpfen dabei an die These der ,grenzenlosen“ Mobilität von Hochqualifizierten an und prüfen diese am Beispiel der nationalen Grenzen der Bundesrepublik. Gefragt wird nach der Strukturierung der Arbeitsmigration von Hochqualifizierten durch diese nationalen Grenzen. Dies wird am Beispiel von Managern in der Wirtschaft und von Medizinern - beides Berufsgruppen, denen in der öffentlichen Debatte eine besonders hohe grenzüberschreitende Mobilität nachgesagt wird (Stichwort ,brain drain') - untersucht. Dabei zeigen die Autoren anhand von eigenen empirischen Studien sowie einer Sekundäranalyse verschiedener geographischer und soziologischer Studien zu grenzüberschreitender Mobilität, dass sich die ,starke‘ Globalisierungsthese nicht aufrechterhalten lässt, sondern nur eine ,schwächere' Fassung: Ihr Fazit ist, ,,dass die internationale Mobilität des hochqualifizierten Personals zwar im Zuge der Globalisierung der Wirtschaft zugenommen hat, aber den Regeln des national geprägten Karrieresystems weiterhin folgt.“ (S. 14) Diesen Befund erklären Pohlmann und Bär unter Rekurs auf organisationssoziologische Theorieangebote.

Heiner Minssen fragt in seinem Beitrag nach dem Zusammenhang von Auslandsentsendungen und Karriere. Er greift dabei die sowohl in der wissenschaftlichen Debatte um einen Formwandel von Karriere als auch in der Praxis weit verbreitete Ansicht auf, dass heute ein Auslandseinsatz für eine erfolgreiche Karriere unverzichtbar sei. Anhand einer Studie zu Auslandseinsätzen in der deutschen chemischen Industrie geht Minssen zunächst der Frage nach, welche Rolle Auslandsentsendungen dort in quantitativer Hinsicht überhaupt spielen. Es zeigt sich, dass der Einsatz von Expatriates eine weit geringere Rolle spielt, als in den Debatten um den vollmobilen, Globalmanager' nahegelegt wird. In einem zweiten Schritt werden dann die Motive, die Erfahrungen und die Karrierefolgen im Zusammenhang mit Auslandseinsätzen der interviewten Expatriates rekonstruiert. Minssen zeigt hier, dass Karriereinteressen zum einen nicht das einzige, häufig nicht einmal das wichtigste Motiv für die Entscheidung für einen Auslandseinsatz sind. Wenn Karriereinteressen eine Rolle spielen, dann im Sinne der ,subjektiven“ Karriere, d. h. der Gestaltung einer subjektiv befriedigenden Berufsbiographie, ohne dass damit zwingend ein positionaler Aufstieg verbunden sein muss. Vielmehr spielen hier arbeitsinhaltliche Ansprüche sowie ,außer-gewöhnliche“ Erfahrungsmöglichkeiten eine Rolle. Es zeigt sich dann ex post aber auch, dass ein Auslandseinsatz tatsächlich die ,objektive Karriere nicht befördert. Die zurückkehrenden Expatriates rücken wieder auf ihrer früheren oder einer vergleichbaren hierarchischen Position in die Zentraleorganisation ein, Karriere im Sinne eines positionalen Aufstiegs machen die meisten nicht. Dies führt dennoch im Allgemeinen nicht zu einem Wechsel der Organisation - die ehemaligen Expatriates bleiben ihrem Unternehmen treu und verbuchen den Auslandseinsatz als jedenfalls interessante und bereichernde Erfahrung. Von der Durchsetzung der boundaryless oder protean career als neuer, nicht mehr organisationsgesteuerter Karriereform kann jedenfalls - so Minssens Fazit - nicht die Rede sein.

Auch für Peter Kels und Uwe Vormbusch beschreiben die genannten Thesen eher die idealtypisierten Anforderungen an und heroisierende Vorstellungen von einem spezifischen Managementtypus als dessen konkrete soziale und biografische Situation. Im Zentrum ihrer Studie zu transnationaler Projektarbeit stehen daher die „Widersprüche und Problemkonstellationen transnationaler Karrieren im Spannungsfeld von Arbeit, 
Biographie und alltäglicher Lebensführung.“ (S. 61) Ihre Studie nimmt die Gruppe der im operativen Management transnationaler Projekte eingesetzten hochqualifizierten Angestellten in den Blick. Die Autoren konstatieren dabei ein „,biografisch in vielfältiger Weise präsentes Beharren auf regionalen und sozialen Bezügen in den Lebensentwürfen und biographischen Bilanzierungen des operativen Managements im globalen Einsatz“(S. 62): Zwar entwickeln die Manager im Auslandseinsatz durchaus die von der ,Globalmanager'-These postulierte Kompetenz zur Überbrückung kultureller und lokaler Unterschiede. Diese Erfahrungen und Kompetenzen bleiben aber gegenüber fortbestehenden Bindungen an Familie, Freunde und einen konkreten ,Ort` sekundär. Hier wird also der These einer Entbettung des grenzüberschreitend tätigen Managements aus lokalen Bezügen empirisch gestützt eine Absage erteilt.

Markus Gottwald und Matthias Klemm untersuchen die ,Globalmanager'-These aus diskurstheoretischer Perspektive und fragen nach deren Funktion. Die Autoren argumentieren in ihrem Beitrag, dass die ,Globalmanager'-These als Antwort auf das ,,steuerungstheoretische Dilemma der Einbeziehung regional verteilter Informationen und Interessen in die Entscheidungsprozesse transnationaler Unternehmen und dem dabei auftretenden Legitimationsproblem der Wahl jener Elemente, Informationen und Perspektiven, die im Unternehmenssinne berücksichtigt werden (sollen) und welche nicht" (S. 78), zu verstehen sei. Die Frage nach der Funktion dieser Reflexionstheorie in transnationalen Unternehmen führt zur Aufdeckung eines grundlegenden Dilemmas, das der Führungskräftenachwuchs in transnationalen Unternehmen ,bearbeiten' muss: Nachwuchsmanager, die eine ,Globalmanager'-Karriere anstreben und deshalb bereit sind, Auslandseinsätze zu absolvieren, müssen einerseits, um solche Einsätze erfolgreich zu bewältigen, sich ,lokalisieren', d. h. mindestens partiell sich die lokalen Perspektiven und das Wissen an ihrem Einsatzort zu eigen machen. Andererseits sind diese je lokalen Perspektiven und Wissensbestände auf der Ebene der Zentrale nicht zu integrieren, da die vielen unterschiedlichen lokalen Perspektiven von der Zentrale aus nicht evaluiert und für die Integration in Konzernstrategien selektiert werden können. Deshalb entwirft die Reflexionstheorie des ,Globalmanager' diesen als ,entbettet', einer eben nicht lokalen, sondern ,abgehobenen“ Rationalität und universellen Wirtschaftlichkeitspostulaten verpflichtet. „So verstanden verweist das Globale am Globalmanager auf den kleinsten gemeinsamen Nenner einer als global bezeichneten Führungs- und Managementkultur, in der Entscheidungen und die Zugehörigkeit zum Kreis der Entscheider durch den Rekurs auf, universelle' Wirtschaftlichkeitsprinzipien begründet werden. Verankert ist diese Form der Rationalisierung weniger in einer, Weltklasse' mit überlegener Einsicht oder in einer gemeinsamen Interessenlage, sondern in den Informations- und Entscheidungsdilemmata transnationaler Wertschöpfung. “ (S. 99)

Vor diesem Hintergrund sind ,starke Thesen", die bereits die Entstehung einer neuen „Weltklasse“ (Kanter 1996) oder transnationalen Bourgeoisie (Sklair 1995) aus ,Globalmanagern“ mit kosmopolitischem Habitus, einem einheitlichen Lebensstil und transnationaler globaler Vernetzung postulieren, höchst kritisch zu bewerten. Alle hier versammelten Beiträge zeigen, dass die Auslandseinsätze der mittleren Fach- und Führungskräfte Episoden in den Berufsbiographien und Karrieren bleiben und dass ,Heimat“ im sozialen wie im räumlich-lokalen Sinne nicht an Bedeutung verliert. Pohlmann und Bär sprechen deshalb von „Internationalisierung light“ und meinen damit, dass zwar im 
Zuge der Transnationalisierung von Wirtschaftsorganisationen Auslandseinsätze von Fach- und Führungskräften häufiger werden, dass daraus aber noch nicht - wie die ,starken' Globalisierungsthesen behaupten - die Herausbildung transnationaler Karrieren auf der Basis globaler Manager-Arbeitsmärkte folgt. Die Emergenz einer Gruppe von vollmobilen, ,entbetteten', überall und nirgends beheimateten ,Globalmanager' ist folgt man den hier versammelten Beiträgen und ihren auf der Basis unterschiedlichen empirischen Datenmaterials und methodischen Vorgehens erzielten Ergebnissen - vorerst nicht zu vermelden.

Neben der Verbreiterung der empirischen Basis für Debatten um Globalisierungsprozesse im Management leisten die hier versammelten Beiträge eine Relativierung und gleichzeitig eine Präzisierung der in diesen Debatten dominierenden Thesen in drei Aspekten:

Erstens kann von einer Ablösung organisationaler Karrieresteuerung durch individuelle Herstellung der eigenen employability und marketability keine Rede sein. Nach wie vor orientieren organisationale Karrieresysteme, in denen sowohl gesellschaftliche als auch organisationale Wertekomplexe institutionalisiert sind (vgl. Pohlmann und Bär), die Managerkarrieren. Eine ,Amerikanisierung' des deutschen Karrieremodells im Sinne einer Tendenz zur transnationalen Allokation von hochqualifizierter Arbeit und Managementtätigkeitt über den Markt und die Herausbildung entsprechender globaler Arbeitsmärkte für Hochqualifizierte und Manager ist nicht zu beobachten (Pohlmann und Bär; Minssen). Ein fundamentaler Wandel der Karrieremuster, wie von den Thesen der boundaryless und protean carreer postuliert, findet also nicht statt. Was allerdings zu beobachten ist, ist eine Umorientierung von der ,objektiven“ (d. h. an positionalem Aufstieg messbaren) zur ,subjektiven ' (d. h. an der Zufriedenheit bezüglich arbeitsinhaltlicher und gestalterischer Ansprüche messbaren) Karriere bei den mittleren Fach- und Führungskräften (vgl. Minssen). Es ist also durchaus ein Wandel ad Karriere zu konstatieren, aber nicht im Sinne der ,forschen“ Thesen der amerikanischen Managementforschung.

Zweitens widerlegen die Beiträge die Vorstellung von der Ort- und Bindungslosigkeit von ,Globalmanagern'. Sie arbeiten vielmehr die soziale und örtliche Einbettung von Managerarbeit und Karrieren heraus. Mit dem Nachweis der Episodenhaftigkeit von Auslandseinsätzen, der fortdauernden Bedeutung von ,Heimat' in einem örtlichen Sinne und der lebensweltlichen Ansprüche, die sich offenbar für die meisten Fach- und Führungskräfte nicht im Rahmen von räumlich entbetteten transnationalen Netzwerken erfüllen lassen, ist auf deutliche Grenzen für die Herausbildung einer supra-lokalen, transnationalen ,Weltklasse“ von ,Globalmanagern' verwiesen. Auch die ,Globalmanager"These muss daher auf der Basis der vorliegenden Beiträge relativiert werden: Zwar entwickeln sich Manager im Rahmen von Auslandseinsätzen oder transnationaler Projektarbeit zu ,Brückenbauern“ und ,Übersetzern', die tatsächlich über die von der ,Globalmanager'-These postulierte interkulturelle Kompetenz und multiperspektivische Haltung verfügen, trotzdem sind sie nolens-volens je lokal verortet - dort wo sie gerade im Einsatz sind. Dieses Ergebnis widerspricht auch der in der postmodernen Theorie vertretenen These von der zunehmenden gesellschaftlichen Bedeutung von Sozialtypen wie dem Flaneur, dem Vagabunden oder dem Nomaden, die - in gewisser, Wahlverwandtschaft' zur ,Globalmanager'-These ebenfalls eine Entbettung aus festen Sozialbezügen postuliert (vgl. Kels und Vormbusch). 
Drittens schließlich zeigen die vorliegenden Beiträge, dass - trotz der angeführten Relativierungen - die ,Globalmanager'-These von organisationaler und gesellschaftlicher Bedeutung ist. Die organisationale Bedeutung liegt dabei aber nicht darin, dass der ,Globalmanager', so wie von Bartlett und Ghoshal (1990) angenommen, die Lösung für die Integrations- und Steuerungsanforderungen transnationaler Unternehmen darstellt, sondern vielmehr - im Sinne des diskurstheoretischen Beitrags von Gottwald und Klemm - darin, dass es sich um eine Problembeschreibung und gleichzeitig um eine Semantik, die als Lösung ausgeflaggt wird, handelt. Und die gesellschaftlichen Folgen der tatsächlich empirisch beobachtbaren, quantitativ und qualitativ zunehmenden Bedeutung von Auslandseinsätzen und transnationaler Kooperation im Management liegen nicht in der Herausbildung globaler Arbeitsmärkte für Hochqualifizierte, in einer Individualisierung und Vermarktlichung von Karrieren jenseits der (Wirtschafts-)Organisation oder gar in der Herausbildung einer transnationalen Bourgeoisie oder kohäsiven Klasse von ,Globalmanagern“, sondern in den Spannungen und Widersprüchen, die sich aus dem normativen Postulat des ,Globalmanagers' als dem Manager transnationaler Unternehmen und den diesen gegenläufigen organisationalen sowie den individuell-lebensweltlichen Ansprüchen ergeben und die die designierten ,Globalmanager' selber auszubalancieren haben. Hier liegt ein noch weitgehend offenes Forschungsfeld für eine gesellschaftstheoretisch ambitionierte Arbeitssoziologie.

Abschließend möchte ich als Herausgeberin all denjenigen danken, die zum Zustandekommen dieses Themenhefts beigetragen haben: der Autorin und den Autoren für die produktive und verlässliche Zusammenarbeit, den Gutachterinnen und Gutachtern der Manuskripte, der Redaktion der ÖZS, insbesondere Franz Ofner, der dieses Heft von der ersten Idee bis zur Publikation helfend und anregend begleitet hat, und nicht zuletzt Karen Meehan (Institut für Soziologie der Universität Klagenfurt) und Simone Claus (Fakultät für Soziologie der Universität Bielefeld), die den Entstehungsprozess des Heftes administrativ begleitet und die Manuskripte ,in Form gebracht' haben.

Bielefeld, im September 2009

\section{Anmerkungen}

1 Der Titel referiert auf Publikationen von Kanter (1996) und Klemm/Popp (2006).

2 „Management am Scheideweg?“, Herbsttagung der Sektion Arbeits- und Industriesoziologie in der Deutschen Gesellschaft für Soziologie, Osnabrück, 9.-10. 10. 2009; „Neue Werte in den Führungsetagen?“, Tagung des Instituts für Soziologie der Universität Heidelberg und des Instituts für Soziologie der Universität Erlangen-Nürnberg, Evangelische Akademie Baden, Bad Herrenalb, 25./26. 9. 2009; Kotthoff/Wagner (2008: „Die Leistungsträger“; vgl. Buchbesprechung in diesem Heft).

3 Ähnlich gelagerte Thesen finden sich auch bei Sklair (1995), Marceau (1989) oder Dahrendorf (2004). 


\section{Literatur}

Altvater, Elmar und Birgit Mahnkopf. 1997. Grenzen der Globalisierung. Ökonomie, Ökologie und Politik in der Weltgesellschaft. Münster: Westfälisches Dampfboot.

Arthur, Michael B. und Denise M. Rousseau. 1996. The Boundaryless Career. A New Employment Principle for a New Organizational Era. Oxford u. a.: Oxford University Press.

Bartlett, Christopher. A. und Sumantra Ghoshal. 1990. Internationale Unternehmensführung. Frankfurt am Main/New York: Campus.

Black, J. Stewart., Hal B. Gregersen, and Mark E. Mendenhall. 1992. Global assignments: successfully expatriating and repatriating international managers. San Francisco: Jossey-Bass.

Boltanski, Luc und Éve Chiapello. 2003. Der neue Geist des Kapitalismus. Konstanz: UVK.

Bossard, Annette B. and Richard B. Peterson. 2005. The Repatriate Experience as Seen by American Expatriates. Journal of World Business 40: 9-28.

Dahrendorf, Ralf. 2004. Der Widerbeginn der Geschichte. Vom Falle der Mauer zum Krieg im Irak. München: Beck.

Faist, Thomas. 2003. The Border-Crossing Expansion of Social Space: Concepts, Questions, and Topics. In: Transnational Social Spaces: Agents, Networks and Institutions, hrsg. Thomas Faist and Eyup Özveren, 1-34. Aldershot: Ashgate.

Faust, Michael, Peter Jauch und Petra Notz. 2000. Befreit und entwurzelt: Führungskräfte auf dem Weg zum ,internen Unternehmer'. München; Mehring: Hampp.

Hall, Douglas. T. 2004. The Protean Career: A Quarter-Century Journey. Journal of Vocational Behavior 65: 1-13.

Hartmann, Michael. 1999. Auf dem Weg zur transnationalen Bourgeoisie? Die Internationalisierung der Wirtschaft und die Internationalität der Spitzenmanager Deutschlands, Frankreichs, Großbritanniens und der USA. Leviathan 27: 113-141.

Hartmann, Michael. 2007. Eliten und Macht in Europa. Ein internationaler Vergleich. Frankfurt/ M.; New York: Campus.

Kanter, Rosabeth M.1996. Weltklasse. Im globalen Wettbewerb lokal triumphieren. Wien: Ueberreuter.

Klemm, Matthias und Michael Popp. 2006: Nomaden wider Willen: Der Expatriate als Handlungstypus zwischen Alltagswelt und objektiver Zweckbestimmung. In: Nomaden, Flaneure, Vagabunden. Wissensformen und Denkstile der Gegenwart, hrsg. W. Gebhardt und R. Hitzler, 126-139. Wiesbaden: VS-Verlag für Sozialwissenschaften.

Kotthoff, Hermann. 1997. Führungskräfte im Wandel der Firmenkultur. Quasi-Unternehmer oder Arbeitnehmer? Berlin: Sigma.

Kotthoff, Hermann und Alexandra Wagner. 2008. Die Leistungsträger. Führungskräfte im Wandel der Firmenkultur - eine Follow-up-Studie. Berlin: Sigma.

Kreutzer, Florian und Silke Roth (Hg.). 2006. Transnationale Karrieren. Biographien, Lebensführung und Mobilität. Wiesbaden: VS-Verlag für Sozialwissenschaften.

Mayrhofer, Wolfgang, Michael Meyer, Johannes Steyrer, Alexander Iellatchitch, Michael Schiffinger, Guido Strunk, Christiane Erten-Buch, Anett Hermann und Christine Mattl: Einmal gut, immer gut? Einflussfaktoren auf Karrieren in ,neuen' Karrierefeldern. Zeitschrift für Personalforschung 16: 392-415.

Marceau, Jane. 1989. A Family Businsess? The Making of an International Business Elite. Cambridge: Cambridge University Press.

Mense-Petermann, Ursula. 2005. Transnationale Kulturalität - Ein konzeptioneller Vorschlag zum Problem der Sozialintegration transnationaler Konzerne am Beispiel der Automobilindustrie. In: Und es fährt und fährt . . . Automobilindustrie und Automobilkultur am Beginn des 21. Jahrhunderts, hrsg. Gert Schmidt, Holger Bungsche, Thilo Heyder und Matthias Klemm, 173-200. Berlin: Sigma. 
Mense-Petermann, Ursula und Gabriele Wagner. 2006. Transnationale Konzerne - Ein neuer Organisationstyp? Wiesbaden: VS-Verlag für Sozialwissenschaften.

Pohlmann, Markus. 2002. Management, Organisation und Sozialstruktur - Zu neuen Fragestellungen und Konturen der Managementsoziologie. In: Managementsoziologie. Themen, Desiderate, Perspektiven, hrsg. Schmidt, Rudi, Hans-Joachim Gergs und Markus Pohlmann, 227-244. München; Mehring: Hampp.

Pries, Ludger. 2008. Die Transnationaliserung der sozialen Welt. Frankfurt a. M.: Suhrkamp.

Schmidt, Rudi, Hans-Joachim Gergs und Markus Pohlmann. 2002. Managementsoziologie. Themen, Desiderate, Perspektiven. München; Mehring: Hampp.

Sklair, Leslie. 1995. Sociology of the Global System, Hemel Hempstead: Prentice Hall/Harvester Wheatsheaf.

Stichweh, Rudolf. 2001. Die Weltgesellschaft - Strukturen eines globalen Gesellschaftssystems jenseits der Regionalkulturen der Welt. Forschung an der Universität Bielefeld 23: 5-10.

Wagner, Gabriele. 2006. Expatriates als Netzwerkarchitekten. In: Transnationale Konzerne - ein neuer Organisationstyp? hrsg. Ursula Mense-Petermann und Gabriele Wagner, 225-247. Wiesbaden: VS-Verlag für Sozialwissenschaften.

Zündorf, Lutz. 1999. Dimensionen weltwirtschaftlicher Vergesellschaftung. Weltmärkte, transnationale Unternehmen und internationale Organisationen. In: Global Players in lokalen Bindungen. Unternehmensglobalisierung in soziologischer Perspektive, hrsg. Eckardt, Andrea., Holm-Detlev Köhler und Ludger Pries, 31-52. Berlin: Sigma.

Ursula Mense-Petermann ist Professorin für Arbeits- und Wirtschaftssoziologie, Fakultät für Soziologie, an der Universität Bielefeld. Forschungsschwerpunkte: Arbeits-, Wirtschafts-, Organisationssoziologie; ökonomische Globalisierung, Transnationalisierungsprozesse. Wichtigste Publikationen: Transnationale Konzerne - ein neuer Organisationstyp? Wiesbaden 2006 (Hg. zusammen mit G. Wagner); Transnationalisierung, Organisation und Kultur. Organisationswissenschaftliche Kulturbegriffe auf dem Prüfstand. In: Berliner Journal für Soziologie, Heft 3, 2006: 393-411; Unternehmen im Transnationalisierungsprozess - Zum Problem der Herstellung de-facto-akzeptierter Entscheidungen in transnationalen Integrationsprozessen. In: Soziale Welt, Heft 4, 2005: 381-397. Adresse: Universität Bielefeld, Fakultät für Soziologie, Postfach 1001 31, D-33501 Bielefeld. 